\title{
Emotional Regulation Activities Moderates the Risk of Depression in People With Sleep Disturbance: Evidence From a Community Health Survey
}

\author{
Jihye Oh, MD, Seung-Chul Hong, MD, PhD \\ Department of Psychiatry, St. Vincent's Hospital, College of Medicine, The Catholic University of Korea, Suwon, Korea
}

Received: November 22, 2021

Revised: December 10, 2021

Accepted: December 20, 2021

Correspondence

Seung Chul Hong, MD, PhD

Department of Psychiatry,

St. Vincent's Hospital,

College of Medicine,

The Catholic University of Korea,

93 Jungbu-daero, Paldal-gu,

Suwon 16247, Korea

Tel +82-31-249-7114

Fax +82-31-248-6758

E-mail hscjohn@hotmail.com

ORCID

Jihye Oh

https://orcid.org/0000-0002-6865-3935

Seung-Chul Hong

https://orcid.org/0000-0003-0828-2906

(c) This is an Open Access article distributed under the terms of the Creative Commons Attribution Non-Commercial License (https://creativecommons.org/licenses/by-nc/4.0) which permits unrestricted non-commercial use, distribution, and reproduction in any medium, provided the original work is properly cited.
Background and Objective It is widely known that poor sleep quality is closely related to depression, but there are limited studies on the mediating factors.

Methods Based on national health survey data, the relationship between sleep quality and depressive symptoms was explored, and structural equations were drawn to determine whether emotional regulation activities, such as regular breakfast, regular exercise, smoking, and drinking contribute to poor sleep quality-induced depression.

Results There was a significant correlation between poor sleep quality and depressive symptoms. Regular breakfast tended to mediate between poor sleep quality and depression the most, followed by smoking, drinking, and regular exercise.

Conclusions Several emotional regulation activities including regular breakfast, can play a protective role in the process leading to poor sleep quality-induced depression. A follow-up study is required to examine the clinical aspect of how regular breakfast can prevent the process of poor sleep quality to depression.

Sleep Med Res 2021;12(2):94-100

Keywords Poor sleep quality; Depression; Mediation analysis; Regular breakfast.

\section{INTRODUCTION}

Sleep is an altered consciousness phenomenon that occurs not only in humans but also across all animal species and is essential in maintaining good health and quality of life [1]. The quality of sleep has a profound effect on mental health, especially on emotional regulation [2]. It is well known that sleep plays a major role in the processing of daily emotions, consequently, poor sleep quality triggers negative emotions and causes emotional dysregulation [3]. Therefore, poor sleep quality is a potential risk factor for the development of mental disorders [2].

A negative stimulus elicits a wide range of corrective responses; however, when the responses fail or are inadequate, negative emotion occurs [4]. Positive psychology believes that self-control can trigger positive emotions [5], and if an individual fails to trigger positive emotions through self-control activities, emotional control difficulties arise. These emotional regulation difficulties are closely related to depression [6]. Examples of human emotional regulation behaviors in positive psychology include eating a hearty meal and exercising [7] while unhealthy emotional regulation behaviors include smoking and drinking alcohol [8].

Sleep problems are core symptoms of depression. Approximately $75 \%$ of depressed patients have insomnia or hypersomnia symptoms, and the sleep problems cause huge distress with a major impact on the quality of life [2]. However, although there is a high correlation between sleep quality and depression, poor sleep quality does not necessarily cause depressive symptoms [9]. For instance, insomnia does not necessarily mean a major depressive disorder, and 
many people with poor sleep quality do not develop mood disorders $[3,10,11]$. We, therefore, assume that several variables mediate the process from poor sleep quality to depression. Among the possible parameters are an individual's biological vulnerability, and an individual's choices of emotion control activities.

Therefore, this study aimed to evaluate the connection between individual emotional self-regulation activities, sleep quality, and depressive symptoms. Using mediation analysis, we analyzed community survey data to investigate if there is a correlation between daily emotion regulation activities (such as eating breakfast, exercise, smoking, and drinking), sleep quality, and depression. We further examined whether daily emotional regulation activities have a mediating effect on the development of depression due to poor sleep quality using a structural equation model.

\section{METHODS}

\section{Study Population and Data Collection}

For this study, we used data from the 2018 Korea Community Health Survey (KCHS), conducted by the Korea Centers for Disease Control and Prevention. The KCHS is a nationwide, cross-sectional survey with a study population from a multi-stage cluster sampling, stratified by primary sample units of civilian, non-institutionalized Korean households by geographic area, age, and gender groups. The survey is conducted annually, and it collects information through face-to-face and a paper-assisted personal interview between interviewers and respondents. The sample size for the KCHS is 900 subjects in each of 253 community units, including 16 metropolitan cities and provinces. Because the population sample is selected using a probability proportional to the sampling methods and, weighted by statisticians who performed post-stratification and considered the non-response rates, samples are representative of the Korean population.

This study included individuals aged 19 years or older. Of the 228340 total survey participants, respondents who did not provide data on their income, employment status, smoking status, alcohol consumption, meal activities, exercise behavior, and scale for sleep quality, or scale for depressive symptoms were excluded. Finally, a total of 89470 eligible participants were included in this study (Fig. 1).

\section{Sleep Quality and Depressive Symptoms}

Sleep quality was measured using the Korean version of the Pittsburg Sleep Quality Index (PSQI-K) which included several sleep-related behaviors; in 2018, PSQI-K was included in the KCHS for the first time. The PSQI is a 19-items self-reported questionnaire that measures the quality and patterns of sleep over a 1-month duration [12]. This study consisted of 7 sleep components: C1, subjective sleep quality; $\mathrm{C} 2$, sleep latency; C3, total sleep duration; $\mathrm{C} 4$, sleep efficiency; $\mathrm{C} 5$, sleep disturbance; $\mathrm{C6}$, use of sleep medication; and C7, daytime dysfunction. The

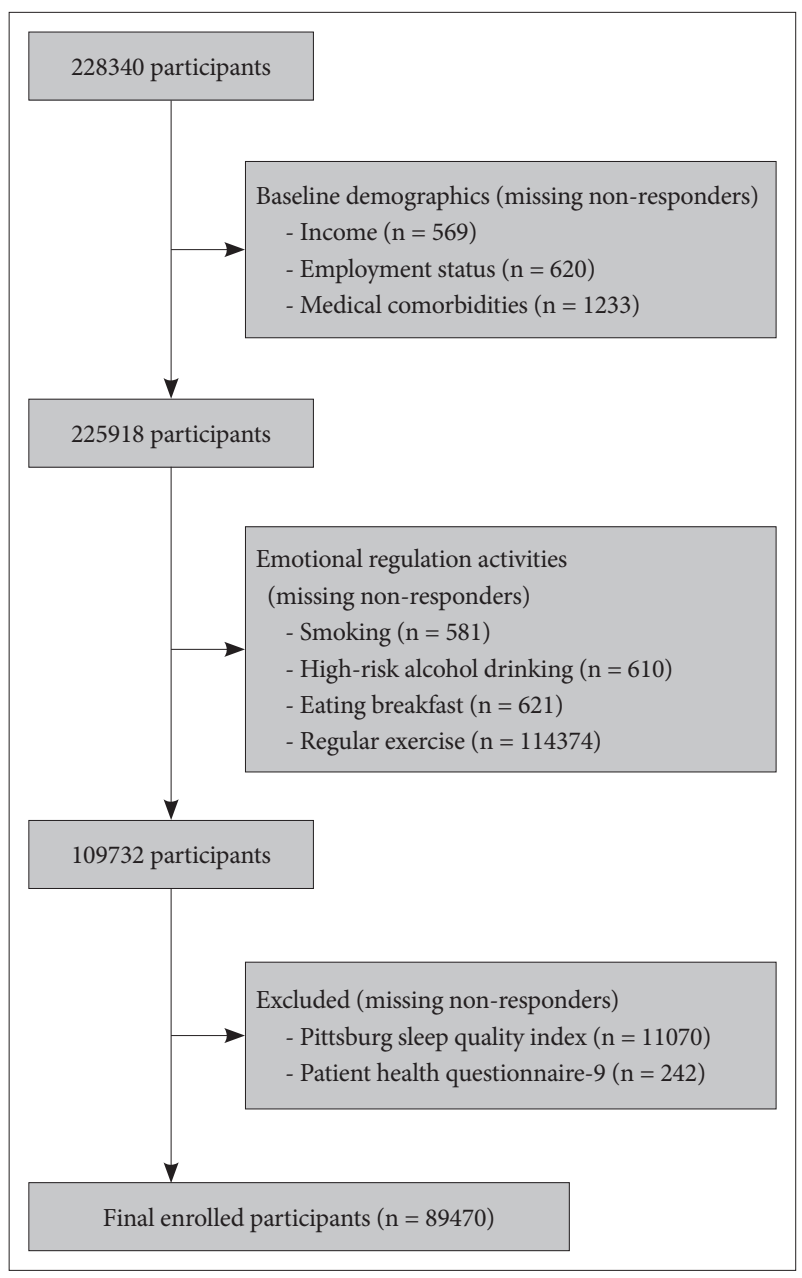

Fig. 1. Flow chart of participants.

PSQI-K has been validated and shown to have adequate reliability [13]. Total PSQI score has been widely used before to measure sleep quality. In this paper, the lower the sleep quality, the higher the total PSQI score, and the higher the sleep quality, the lower the total PSQI score, the higher total PSQI score was regarded as poor sleep quality.

Depressive symptoms were measured using the Patient Health Questionnaire-9 (PHQ-9) that has been widely used in population-based studies because it's self-reporting and simple. The Korean version of PHQ-9 has also been validated and shown high reliability [14].

\section{Emotional Regulation Activities}

The emotional regulation activities measured in this study were as follows: regular breakfast, regular exercises, smoking, and alcohol drinking. The number of days that people had eaten breakfast in the previous week among people who reported eating their meals regularly was used to define regular breakfast. Among the participants who answered to exercise behavior, the most recent one-month regular exercise was defined as regular exercise. We defined alcohol drinking-related behavior with a 
subscale of The Korean version of Alcohol Use Disorders Identification Test to measure how much alcohol was consumed in the most recent month. The variable of smoking was classified as smoking every day, occasionally smoking, and not smoking.

\section{Statistical Analysis}

Since the data from KCHS were collected using stratified, clustered, and systematic sampling, complex survey dataset analysis methods were used while following the guidelines of KCHS analysis, which were published by the Korea Centers for Disease Control and Prevention [15]. Descriptive statistics were used to identify the participants' baseline characteristics. Logistic regression analysis was done, and the receiver operating characteristic (ROC) curve was drawn to evaluate the feasibility of sleep quality in predicting depressive symptoms. To explore the effects of emotion regulation activities that mediate the link between sleep quality and depressive symptoms, correlation analyses were done. We used statistical methods in a way that created structural equation methods and validated this model. Since there are many missing values, the maximum likelihood with the missing value method is used for estimation. We used a different stepping algorithm in nonconcave regions for maximization. Optimization was done by 300 maximum iterations. Covariance structure used the following: sex, age, household income level, employment status, and current medical comorbidity which could affect sleep quality (diabetes mellitus, hypertension, cerebrovascular disease, asthma, allergic rhinitis, atopic dermatitis). Differences were considered statistically significant at ap-value less than 0.05. Statistical analyses were performed using STATA version 16 (STATA Corp., College Station, TX, USA).

This study was approved by the Institutional Review Board of St. Vincent's Hospital in Korea (VC21ZASI0245).

\section{RESULTS}

\section{Baseline Participants' Characteristics}

The demographics and baseline characteristics of participants are presented in Table 1. The mean age of the participants was $54.52 \pm 17.58$ years, and $44.78 \%$ of the participants were male. The average monthly household income of the participants was $46.88 \pm 35.84$ million won, and $61.58 \%$ of participants were currently employed. $32.68 \%$ of the participants had medical comorbidities. $16.01 \%$ said they smoke every day, $1.32 \%$ said they smoke occasionally and $82.66 \%$ said they don't smoke. $32.55 \%$ of the patients said they didn't drink alcohol, $16.73 \%$ said they drink less than once a month, $8.91 \%$ said they drink twice or four times a month, $19.86 \%$ said they drink two to three times a week, and 8.14\% said they drink more than four times a week. Among those who said they eat regularly, $11.33 \%$ said they had never eaten breakfast in the past week, $11.33 \%$ said they had eaten once, $3.51 \%$ said they had eaten twice, $4.44 \%$ said they had eaten twice,
$2.50 \%, 5.65 \%$, and $1.46 \%$ said they had eaten six times. Of the participants who responded to exercise behavior, $39.20 \%$ reported exercising regularly.

\section{Correlation between Sleep Quality and Depressive Symptoms}

When the logistic regression analysis was performed to see how well the total score of PSQI predicted the degree of depressive symptoms measured by PHQ-9, the odds ratio was 1.40 (95\% confidential interval [CI] 1.40-1.41, p < 0.001). Based on these results the ROC curve was drawn, and the area under the ROC curve was measured at 0.6690, which is shown in Fig. 2.

\section{Correlations between Different Types of Emotional Regulation Activities, Sleep Quality, and Depressive Symptoms}

Prior to drawing the Structural Equation Model, Pearson cor-

Table 1. Baseline characteristics of participants

\begin{tabular}{|c|c|}
\hline Characteristics & Value \\
\hline \multicolumn{2}{|l|}{ Sex } \\
\hline Male & $40069(44.78)$ \\
\hline Female & $49401(55.22)$ \\
\hline Age (year) & $54.52 \pm 17.58$ \\
\hline \multicolumn{2}{|l|}{ Employment status } \\
\hline Employed & $55093(61.58)$ \\
\hline Not employed & $34377(38.42)$ \\
\hline Monthly household income (million won) & $46.88 \pm 35.84$ \\
\hline \multicolumn{2}{|l|}{ Medical comorbidities } \\
\hline Presence & $29241(32.68)$ \\
\hline Absence & $60229(67.32)$ \\
\hline \multicolumn{2}{|l|}{ Smoking } \\
\hline Yes & $15511(17.34)$ \\
\hline No & $73959(82.66)$ \\
\hline \multicolumn{2}{|l|}{ Drinking } \\
\hline Not drinking & $29125(32.55)$ \\
\hline Low-to-moderate-risk drinking & $40710(45.50)$ \\
\hline High-risk drinking & $19635(21.95)$ \\
\hline \multicolumn{2}{|l|}{ Regularly eating breakfast } \\
\hline Yes & $63853(71.37)$ \\
\hline No & $25617(28.63)$ \\
\hline \multicolumn{2}{|l|}{ Regular exercise } \\
\hline Yes & $35072(39.20)$ \\
\hline No & $54398(60.80)$ \\
\hline Total score of PSQI & $6.69 \pm 2.01$ \\
\hline Total score of PHQ-9 & $2.2 \pm 3.29$ \\
\hline
\end{tabular}

Data are presented as mean \pm standard deviation or $\mathrm{n}(\%)$. PSQI, Pittsburg Sleep Questionnaire Index; PHQ-9, Patient Health Questionnaire-9. 
relation analysis was performed to evaluate the association between each variable, and the results are shown in Table 2. Emotion regulation activities were significantly correlated with PSQI total scores but were not significantly correlated with PHQ-9 total scores.

The structural equation model was drawn to evaluate how emotional regulation activities mediated between sleep quality and depressive symptoms, and the results are shown in Fig. 3 and Table 3. The direct effects of the total PSQI scores on each emotional control behavior are as follows: the correlation between the total score of PSQI and eating breakfast was 0.11 (95\% CI, 0.10-0.11), the effect for regular exercise was 0.05 (95\% CI, 0.05-0.05), the effect for smoking was 0.06 (95\% CI, 0.060.06). Between PSQI and PHQ-9, the mediating effect of eating breakfast was -0.53 (95\% CI, -0.56--0.51) and the mediating effect of regular exercise was -0.09 ( $95 \%$ CI, $-0.12--0.05$ ). The mediating effects between PSQI and PHQ-9 of smoking and alcohol drinking were -0.21 (95\% CI, -0.26--0.17) and -0.16 (95\% CI, -0.17--0.15), respectively. All the results were significant with a p-value $<0.0001$.

\section{DISCUSSION}

This study aimed to identify the role of everyday emotional regulation activities in the development of depressive symptoms

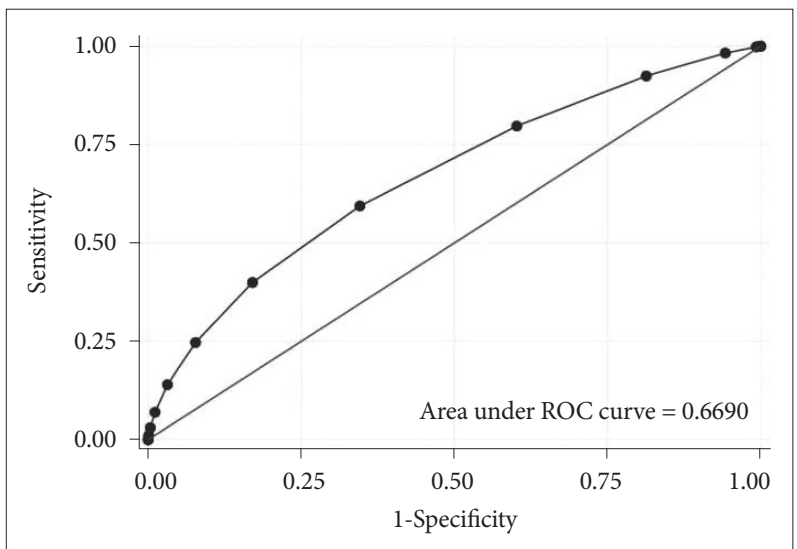

Fig. 2. ROC curve that the total score of Pittsburg Sleep Questionnaire Index is how well predict the total score of Patient Health Questionnaire-9. ROC, receiver operating characteristic. as a result of poor sleep quality. Based on our results, we found emotional regulation activities to be a potent mediator between sleep quality and depression.

Further, there was a significant correlation between PSQI total score and PHQ-9 total score, but it was shown that ROC under the area was 0.66 and poor sleep quality predicted only about $66 \%$ of the depressive symptoms. This is consistent with the existing report that although sleep disturbance is one of the core symptoms of depression, poor sleep quality does not necessarily mean major depression.

The positive correlation between the total PSQI score and emotion regulation activities indicated a correlation between poor sleep quality and regular breakfast eating habits, that is, people try to eat breakfast more regularly to regain regularity in life after experiencing poor sleep quality. In the same context, the tendency to exercise more regularly correlated the poorer sleep quality, although the correlation coefficient was not statistically significant.

The study found that poor sleep quality increases the frequency of alcohol use, and this is consistent with the existing study results $[16,17]$. However, emotion regulation activities alone do not seem to directly correlate with depressive symptoms. Previous studies on the relationship between alcohol/nicotine use and depression showed that there was no correlation, similarly, in this study, there was no correlation between the degree of de-

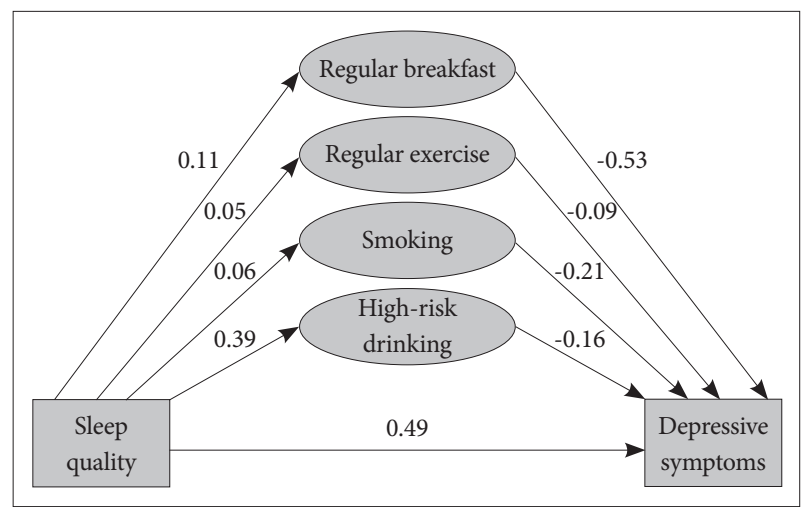

Fig. 3. Structural Equation Modeling for the relationship between sleep quality, depression, and emotional regulation activities. Sleep quality was measured through the total score of Pittsburg Sleep Questionnaire Index, and depressive symptoms were measured through Patient Health Questionnaire-9. All mediation coefficients have significant $p$-values $(p<0.05)$.

Table 2. Correlation among different types of emotional regulation activities, sleep quality, and depressive symptoms

\begin{tabular}{|c|c|c|c|c|}
\hline \multirow{2}{*}{ Emotional regulation activities } & \multicolumn{2}{|c|}{ Correlation with total score of PSQI } & \multicolumn{2}{|c|}{ Correlation with total score of PHQ-9 } \\
\hline & Coefficient & $\mathrm{p}$-value & Coefficient & p-value \\
\hline Smoking & -0.0127 & $<0.0001$ & 0.0030 & 0.1566 \\
\hline Alcohol drinking & 0.0353 & $<0.0001$ & 0.0016 & 0.4601 \\
\hline Eating breakfast & 0.0112 & $<0.0001$ & -0.0006 & 0.7671 \\
\hline Regular exercise & -0.0241 & $<0.0001$ & 0.0035 & 0.2406 \\
\hline
\end{tabular}

PSQI, Pittsburg Sleep Questionnaire Index; PHQ-9, Patient Health Questionnaire-9. 


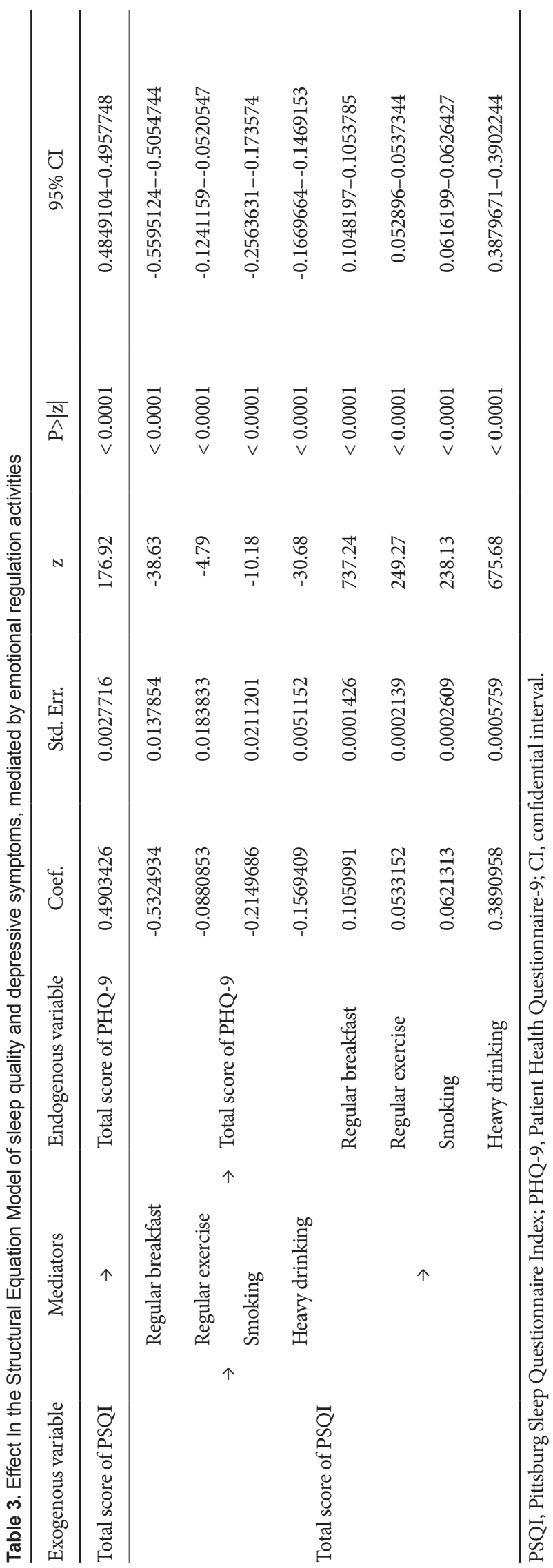

pression and frequency of alcohol and nicotine use.

On the other hand, the application of the structural equation model of the relationship showed that emotion regulation activities had a protective effect on the process of going from poor sleep quality to depression. Eating breakfast was found to prevent depression to a very high degree, and regular exercise had the least protective effect on depression, unexpectedly. Meanwhile, smoking and alcohol drinking have also been shown to have antagonistic effects on depressive symptoms, this is consistent with previous studies and our clinical experience of increased smoking and drinking levels when experiencing negative mood associated with depressive symptoms $[18,19]$.

This study demonstrates that routine emotion regulation activities are protective of the sleep disturbance to depression process. Eating breakfast regularly seems to have a preventive effect on the process that leads to major depression in patients with poor sleep quality. Based on the results of this study, there was a positive correlation between poor sleep quality, and regular breakfast eating habits, which could prevent depression despite poor sleep quality; several other studies have also concluded that skipping breakfast has a high correlation with depression, and regular breakfast prevents depression [20-26]. This can be termed as part of human self-regulatory adaptive behavior for preventing depression.

Interestingly, our study results confirm our assumption that people with poor sleep quality will increase alcohol consumption or drink alcohol to relieve their negative emotion study. A similar trend is also observed in smoking, and several studies have shown that smoking and drinking can be a kind of emotional control behavior for overcoming depressed moods [2731]. However, addictive substances such as alcohol and nicotine harm health, making it difficult to recommend them as a means of relieving negative emotions. Therefore, based on the findings, real-world clinicians can advise patients that eating breakfast and exercising regularly could prevent depression.

Although this paper does not publish its results, it has shown that these emotion regulation activities have little effect in preventing suicidal ideation. Therefore, our daily emotion regulation activities measured in this paper are not absolute depressive symptoms prevention approaches and psychiatric intervention is essential for patients with suicidal ideation who visit the primary clinic.

There are many limitations to this study. First, there may be a reporting bias because it was a survey study based on self-reporting. Furthermore, biological effects such as family history and bipolarity that can affect depression must be considered; this could not be considered in this study. There is also a large amount of missing data even though the interviews were conducted by trained interviewers. Since the survey was conducted by laymen who did not have clinical experience in sleep medicine, rather than experienced interviewers who specialized in measuring PSQIs, there may be errors such as confusion in the morning/ 
afternoon. Therefore, although the data were double-checked and the time was corrected, the data accuracy may be low. In addition, education levels were excluded from the covariate, which was thought to be a factor predicting education levels due to the low level of education and the high young population in Korea for decades. In addition, since the study is not aimed at accurate diagnosis and medical records of actual patients, there are many clinical application limitations. Therefore, verifying the findings through actual clinical trials will be necessary.

Nevertheless, to the best of our knowledge, this is the first study to investigate how daily emotion regulation activities correlate with sleep quality and depressive systems. Since this study is a large-scale epidemiological study based on community samples, the findings of this study can be considered as a relatively good reflection of the population.

In conclusion, daily emotional regulation activities, especially eating breakfast regularly, have a protective effect on the process of developing depressive symptoms in patients with poor quality. We plan to conduct further studies on how such emotion regulation activities can prevent depressive symptoms.

\section{Availability of Data and Material}

The datasets generated or analyzed during the study are available from the corresponding author on reasonable request.

\section{Author Contributions}

Conceptualization: Oh J. Data curation: Oh J. Formal analysis: Oh J. Investigation: Oh J. Methodology: Oh J. Project administration: Oh J. Supervision: Hong SC. Validation: Oh J. Visualization: Oh J. Writingoriginal draft: Oh J. Writing — review \& editing: Oh J, Hong SC.

\section{Conflicts of Interest}

The authors have no potential conflicts of interest to disclose.

\section{Funding Statement}

None.

\section{Acknowledgements}

For this study, we used the Korea Community Health Survey data, conducted by the Korea Centers for Disease Control and Prevention.

\section{REFERENCES}

1. Murillo-Rodriguez E, Arias-Carrion O, Zavala-Garcia A, Sarro-Ramirez A, Huitron-Resendiz S, Arankowsky-Sandoval G. Basic sleep mechanisms: an integrative review. Cent Nerv Syst Agents Med Chem 2012; 12:38-54.

2. Kahn M, Sheppes G, Sadeh A. Sleep and emotions: bidirectional links and underlying mechanisms. Int J Psychophysiol 2013;89:218-28.

3. Ewing DL, Manassei M, Gould van Praag C, Philippides AO, Critchley HD, Garfinkel SN. Sleep and the heart: interoceptive differences linked to poor experiential sleep quality in anxiety and depression. Biol Psychol 2017;127:163-72.

4. Markarian SA, Pickett SM, Deveson DF, Kanona BB. A model of BIS/ BAS sensitivity, emotion regulation difficulties, and depression, anxiety, and stress symptoms in relation to sleep quality. Psychiatry Res 2013; 210:281-6.

5. Min JA, Lee CU, Chae JH. Resilience moderates the risk of depression and anxiety symptoms on suicidal ideation in patients with depression and/or anxiety disorders. Compr Psychiatry 2015;56:103-11.

6. Halahakoon DC, Kieslich K, O’Driscoll C, Nair A, Lewis G, Roiser JP. Reward-processing behavior in depressed participants relative to healthy volunteers: a systematic review and meta-analysis. JAMA Psychiatry 2020;77:1286-95.

7. Swerdlow BA, Pearlstein JG, Sandel DB, Mauss IB, Johnson SL. Maladaptive behavior and affect regulation: a functionalist perspective. Emotion 2020;20:75-9.

8. Albert KM, Newhouse PA. Estrogen, stress, and depression: cognitive and biological interactions. Annu Rev Clin Psychol 2019;15:399-423.

9. Kim JM, Stewart R, Kim SW, Kim SY, Bae KY, Kang HJ, et al. Physical health and incident late-life depression: modification by cytokine genes. Neurobiol Aging 2013;34:356.e1-9.

10. Lee S, Kim JH, Chung JH. The association between sleep quality and quality of life: a population-based study. Sleep Med 2021;84:121-6.

11. Fatima Y, Bucks RS, Mamun AA, Skinner I, Rosenzweig I, Leschziner $\mathrm{G}$, et al. Sleep trajectories and mediators of poor sleep: findings from the longitudinal analysis of 41,094 participants of the UK Biobank cohort. Sleep Med 2020;76:120-7.

12. Buysse DJ, Reynolds CF 3rd, Monk TH, Berman SR, Kupfer DJ. The Pittsburgh sleep quality index: a new instrument for psychiatric practice and research. Psychiatry Res 1989;28:193-213.

13. Sohn SI, Kim DH, Lee MY, Cho YW. The reliability and validity of the Korean version of the Pittsburgh sleep quality index. Sleep Breath 2012; 16:803-12.

14. Seo JG, Park SP. Validation of the Patient Health Questionnaire-9 (PHQ9) and PHQ-2 in patients with migraine. J Headache Pain 2015;16:65.

15. Lee SY, Ju YJ, Lee JE, Kim YT, Hong SC, Choi YJ, et al. Factors associated with poor sleep quality in the Korean general population: providing information from the Korean version of the Pittsburgh sleep quality index. J Affect Disord 2020;271:49-58.

16. Koob GF, Colrain IM. Alcohol use disorder and sleep disturbances: a feed-forward allostatic framework. Neuropsychopharmacology 2020;45: 141-65.

17. Reid-Varley WB, Ponce Martinez C, Khurshid KA. Sleep disorders and disrupted sleep in addiction, withdrawal and abstinence with focus on alcohol and opioids. J Neurol Sci 2020;411:116713.

18. Fluharty M, Taylor AE, Grabski M, Munafò MR. The association of cigarette smoking with depression and anxiety: a systematic review. Nicotine Tob Res 2017;19:3-13.

19. Aoyama M, Sakaguchi Y, Fujisawa D, Morita T, Ogawa A, Kizawa Y, et al. Insomnia and changes in alcohol consumption: relation between possible complicated grief and depression among bereaved family caregivers. J Affect Disord 2020;275:1-6.

20. Dashti HS, Merino J, Lane JM, Song Y, Smith CE, Tanaka T, et al. Genome-wide association study of breakfast skipping links clock regulation with food timing. Am J Clin Nutr 2019;110:473-84.

21. Zahedi H, Djalalinia S, Sadeghi O, Zare Garizi F, Asayesh H, Payab M, et al. Breakfast consumption and mental health: a systematic review and meta-analysis of observational studies. Nutr Neurosci 2020 Dec 14 [Epub]. https://doi.org/10.1080/1028415X.2020.1853411

22. Ferrer-Cascales R, Sánchez-SanSegundo M, Ruiz-Robledillo N, Albaladejo-Blázquez N, Laguna-Pérez A, Zaragoza-Martí A. Eat or skip breakfast? The important role of breakfast quality for health-related quality of life, stress and depression in Spanish adolescents. Int J Environ Res Public Health 2018;15:1781.

23. Lee SA, Park EC, Ju YJ, Lee TH, Han E, Kim TH. Breakfast consumption and depressive mood: a focus on socioeconomic status. Appetite 2017;114:313-9.

24. Miki T, Eguchi M, Kuwahara K, Kochi T, Akter S, Kashino I, et al. Breakfast consumption and the risk of depressive symptoms: the $\mathrm{Fu}$ rukawa nutrition and health study. Psychiatry Res 2019;273:551-8.

25. Milajerdi A, Keshteli AH, Esmaillzadeh A, Roohafza H, Afshar H, Adibi P. Breakfast consumption in relation to lowered risk of psychological disorders among Iranian adults. Public Health 2019;167:152-8. 
26. Chang ZS, Boolani A, Conroy DA, Dunietz T, Jansen EC. Skipping breakfast and mood: the role of sleep. Nutr Health 2021;27:373-9.

27. Liu Y, Li H, Li G, Kang Y, Shi J, Kong T, et al. Active smoking, sleep quality and cerebrospinal fluid biomarkers of neuroinflammation. Brain Behav Immun 2020;89:623-7.

28. Fluharty M, Taylor AE, Grabski M, Munafò MR. The association of cigarette smoking with depression and anxiety: a systematic review. Nicotine Tob Res 2017;19:3-13.
29. Rubin LF, Haaga DAF, Pearson JL, Gunthert KC. Depression as a moderator of the prospective relationship between mood and smoking. Health Psychol 2020;39:99-106.

30. Boden JM, Fergusson DM. Alcohol and depression. Addiction 2011; 106:906-14.

31. Truong MK, Berger M, Haba-Rubio J, Siclari F, Marques-Vidal P, Heinzer R. Impact of smoking on sleep macro- and microstructure. Sleep Med 2021;84:86-92. 